\title{
PREVENTION AND PROPHYLAXIS OF CONGENITAL DEAFNESS
}

\author{
S. Petrova, D. Georgieva, A.Vlaykov \\ Department of Otorhinolaryngology, Medical Faculty, Trakia University, Stara Zagora, \\ Bulgaria
}

\begin{abstract}
Good hearing is a prerequisite for normal mental and speech development while in adults hearing loss is related to limited hearing and communication difficulties. Hearing may be reduced, mild, moderate, medium and heavy.

The objective is to inform people with congenital deafness on ways and methods of treatment. This methods are: Hearing aids, Surgical techniques, Stepedektomiya, Tympanoplasty, Transplant auditory bones and more, Cochlear implant

The surgical implantation is only the first step in helping children with congenital deafness. In the first years after the surgical intervention the child needs intensive auditory and speech training which are of significant importance.

Deafness depends on whether there is a dominant gene. Diagnosis is set on grounds of the anamnesis and the objective study.
\end{abstract}

Key words: congenital deafness, cochlear implantation, hearing impaired, prevention and prophylaxis.

\section{INTRODUCTION}

The children acquire the ability to speak by emulating adults talk. Good hearing is a prerequisite for normal mental and speech development while in adults hearing loss is related to limited hearing and communication difficulties. Hearing may be reduced, mild, moderate, medium and heavy. There is also the complete hearing loss (1).

In children with inborn hearing loss, aplasia (underdevelopment) and degeneration of the organ of Corti is seen. There might be malformations in the outer and middle ear. Acquired hearing loss is also related with aplastic degenerative changes in the organ of Corti or the auditory nerve.

Inborn deafness is found in people where the hearing loss is a result of (2): Fetal damage (prenatal stage); Absent pinna or outer ear; coalescence of the ear canal, absence of the three bones, etc; Damaged part of the inner ear (cochlear damage), after rubeola in the mother during the first 12-16 weeks of the pregnancy; Disorders around and at the time of birth (perinatal stage); Premature birth; Anoxia; Infant jaundice; Rh incompatibility-in parents with no hearing loss; As a dominant trait in deaf parents; Bilateral congenital hearing loss is an inborn loss of hearing in both ears present at birth. Inborn hearing loss can be inherited in an autosomal dominant, autosomal recessive, or $\mathrm{X}$-linked manner.

Bilateral congenital deafness can be inherited or caused by other factors such as the Down Syndrome, Usher Syndrome, Treacher Collins Syndrome (TCS), Crouzon Syndrome, Alport Syndrome, prenatal infections and diseases (rubeola, morbili, cytomegalovirus, herpes simplex virus infection, toxoplasmosis, syphilis, mumps), influence of teratogenic factors during pregnancy (alcohol and drug abuse, nicotine, medications, exposure to toxic substances, exposure to radiation), Rh-related complications, hypoxia, hyperbilirubinemia, a mother with diabetes, premature birth, inborn defects in the structure of the auditory apparatus, earwax or fluid build-up, raptured eardrum, meningitis, otosclerosis, Ménière's disease, albinism, etc. 
Split hand/split foot-deafness is an inborn malformation characterized by bilateral congenital deafness and limb malformation.

Approximately one in every 1000 to 2000 babies is born deaf.

\section{OBJECTIVE}

The objective is to raise people's awareness of inborn deafness, its treatment methods and its effect onto their quality of life.

\section{MATERIALS AND METHODS}

Hearing aids; Surgical techniques; Stapedectomy; Tympanoplasty; Cochlear implant; Auditory ossicle transplantation; Auditory evoked potentials- measured is the brain's electrical activity as a result of sound stimuli; Diagnostic imaging; Bone X-ray projection; Computed Tomography (CT, scanner); Magnetic resonance imaging (MRI); Vibration testing;

The most effective is the Cochlear Implant System.

\section{COCHLEAR IMPLANT SYSTEM}

The Cochlear Implant System is the most modern achievement of the microelectronics and medicine for stimulation the auditory perception of people with profound sensorineural hearing loss. This auditory prosthesis is designed to improve the auditory sensations by electrical stimulation of the auditory nerve. It is designed for people who are unable to receive adequate speech information with the standard hearing aids.

Cochlear implant systems are successfully used by both children and adults, regardless of the fact whether they have been born deaf or the hearing loss occurs later.

The cochlear implant system has an outer and inner part. The outer part comprises of: speech processor, battery box and transmission coil. The inner part comprises of: implant, active and supporting electrode.

All the electronic parts of the implant are in an air-sealed box. The implant is implanted into the bone behind the ear. The implant is bacteria-free. The energy required to power the implant is provided by the external speech processor and transmitted to the implant together with the signal through the skin.

The active electrode with the contact pairs is placed in the cochlea. It has an electrical connection with the implant. The stimulating contact points are placed onto the active electrode in a way allowing stimulation of the auditory nerve at different places.

The supporting electrode is necessary for closing the electric circuit. It is placed under a muscle behind the ear.

\section{FUNCTION OF COCHLEAR IMPLANT SYSTEM}

The sound waves are captured by a small microphone and transformed into electric signals; The speech processor transforms the electric signals into electric impulses with a specific shape depending on the specific coding speech strategy; The coded signal is sent via cable to the transmission coil; The transmission coil sends the signal and the needed power through the skin to the implant. Using the contact pairs of the active electrode, the transmitted electrical signals stimulate the auditory nerve at different places in the cochlea; As a result, the auditory nerve generates the so called action potentials and sends them to the brain.

The brain receives the action potentials generated by the auditory nerve and reads them as sounds.

\section{RESULTS}

Not every person suffering from a hearing loss can benefit from the cochlear implant. A team of a surgeon and an audiologist defines whether the patient is a suitable candidate. The surgical implantation is only the first step in helping children with congenital deafness. In the first years after the surgical intervention the child needs intensive auditory and speech training which are of significant importance. Without them the child is unable to benefit from the newly acquired sense (hearing), as well as unable to get to know how to use speech when communicating with other people. The speech processor of the implant is programmed in accordance with the needs and desires of the patient and in young children-according the opinion and observations of their parents and rehabilitators. Regular settings of the implant in the first months after its activation help the patient get used to the new sound and benefit from it to a maximum extent.

The aim of the study is the timely cochlear implantation, i.e. at the age of 6 months. By the help of the implant, babies with inborn hearing 
loss shall be able to hear the sounds and shall be able to start talking at the same time with his peers. This medical intervention is extremely useful for child's further development-both mental and physical.

\section{CONCLUSION}

Half of all cases of deafness and hearing loss are hereditary.

Deafness depends on whether there is a dominant gene (4). Diagnosis is set on grounds of the anamnesis and the objective study.

Treatment takes place by prosthetic correction of the hearing and hearing rehabilitation. Treatment is surgical since medication-based treatment is impossible.

\section{REFERENCES}

1. Deafness - results and factors (24.02.2010)

2. Dimov. D, Georgiev. G, learning Ear, nose and throat diseases, p.p.150-170, 1998

3. Deafnes to children http://www.lolov.com/frames/container/info/f aq/ear/deca.html (July, 2005)

4. Congenital deafnes- symptoms http://medpedia.framar.bg/\%D1\%81\%D0\%B $8 \% \mathrm{D} 0 \% \mathrm{BC} \% \mathrm{D} 0 \% \mathrm{BF} \% \mathrm{D} 1 \% 82 \% \mathrm{D} 0 \% \mathrm{BE} \% \mathrm{D}$ $0 \% \mathrm{BC} \% \mathrm{D} 0 \% \mathrm{~B} 8 / \% \mathrm{D} 0 \% \mathrm{~B} 3 \% \mathrm{D} 0 \% \mathrm{BB} \% \mathrm{D} 1 \%$ $83 \% \mathrm{D} 1 \% 85 \% \mathrm{D} 0 \% \mathrm{BE} \% \mathrm{D} 1 \% 82 \% \mathrm{D} 0 \% \mathrm{~B} 0$ (12.08.2011) 